\title{
To drill or not to drill, that is the question: nonsurgical treatment of chronic subdural hematoma in the elderly. A systematic review
}

\author{
Alba Scerrati, MD, ${ }^{1,2}$ Jacopo Visani, MD, ${ }^{2}$ Luca Ricciardi, MD, ${ }^{3}$ Flavia Dones, MD, ${ }^{2}$ \\ Oriela Rustemi, MD, ${ }^{4}$ Michele Alessandro Cavallo, MD, ${ }^{1,2}$ and Pasquale De Bonis, MD, PhD ${ }^{1,2}$ \\ 'Department of Morphology, Anatomy and Experimental Medicine, University of Ferrara; ${ }^{2}$ Department of Neurosurgery, \\ Sant'Anna University Hospital, Ferrara; ${ }^{3}$ Department of Neurosurgery, Pia Fondazione di Culto e Religione Cardinal G. Panico, \\ Tricase, Lecce; and ${ }^{4}$ Department of Neurosurgery, San Bortolo Hospital, Vicenza, Italy
}

\begin{abstract}
OBJECTIVE Chronic subdural hematoma (CSDH) is one of the most common neurosurgical pathologies, typically affecting the elderly. Its incidence is expected to grow along with the aging population. Surgical drainage represents the treatment of choice; however, postoperative complications and the rate of recurrence are not negligible. For this reason, nonsurgical alternatives (such as middle meningeal artery embolization, steroids, or tranexamic acid administration) are gaining popularity worldwide and need to be carefully evaluated, especially in the elderly population.

METHODS The authors performed a systematic review according to PRISMA criteria of the studies analyzing the nonsurgical strategies for CSDHs. They collected all papers in the English language published between 1990 and 2019 by searching different medical databases. The chosen keywords were "chronic subdural hematoma," "conservative treatment/management," "pharmacological treatment," "non-surgical," "tranexamic acid," "dexamethasone," "corticosteroid," "glucocorticoid," "middle meningeal artery," "endovascular treatment," and "embolization."
\end{abstract}

RESULTS The authors ultimately collected 15 articles regarding the pharmacological management of CSDHs matching the criteria, and 14 papers included the endovascular treatment.

CONCLUSIONS The results showed that surgery still represents the mainstay in cases of symptomatic patients with large CSDHs; however, adjuvant and alternative therapies can be effective and safe in a carefully selected population. Their inclusion in new guidelines is advisable.

https://thejns.org/doi/abs/10.3171/2020.7.FOCUS20237

KEYWORDS chronic subdural hematoma; tranexamic acid; dexamethasone; pharmacotherapy; middle meningeal artery; endovascular treatment; embolization

$\mathrm{C}$ HRONIC subdural hematoma $(\mathrm{CSDH})$ represents one of the most common neurosurgical disorders, with an estimated incidence of approximately 58 per 100,000 per year among people older than 70 years. ${ }^{1}$ Its incidence has been progressively increasing along with life expectancy, especially in high-income countries..$^{2-4}$ In the elderly population, often burdened by multiple and severe comorbidities, CSDH appears to consistently impact patients' quality of life, eventually determining poor prognosis due to immobilization. Surgery has always been considered as a gold standard treatment for CSDH because it is a relatively safe and effective first-line management option providing potential health cost savings. ${ }^{5}$ Several meta-analyses and systematic reviews $\mathrm{s}^{5,6}$ evaluated the results of different surgical procedures: no differences were found between twist drill or burr hole, whereas the benefit of postoperative drainage has been clearly demonstrated, as has the use of irrigation.

Although surgical evacuation of CSDH has been widely considered as a straightforward and safe procedure, the recurrence rate is relatively high, long-term clinical outcomes for elderly patients remain poor, and surgical morbidity and mortality are not negligible. ${ }^{7}$ Accordingly, there is an increasing interest in nonsurgical alternatives,

ABBREVIATIONS $\mathrm{CSDH}=$ chronic subdural hematoma; $\mathrm{DX}=$ dexamethasone; $\mathrm{MMAE}=$ middle meningeal artery embolization; $\mathrm{RCT}=$ randomized controlled trial; TXA = tranexamic acid. 
although no comparative studies are currently available. This study aimed to systematically review the pertinent literature on nonsurgical management options for CSDH in the elderly population.

\section{Methods}

The present study consists of a systematic review of the international medical literature conducted according to the PRISMA (Preferred Reporting Items for Systematic Reviews and Meta-Analyses) guidelines. ${ }^{8}$ The PRISMA checklist is detailed in Moher et al. ${ }^{8}$

Three different medical databases (PubMed, Scopus, and Cochrane Library) were selected for our research. The search terms were "chronic subdural hematoma," "conservative treatment/management," "pharmacological treatment," "non-surgical," "tranexamic acid," "dexamethasone," "corticosteroid," "glucocorticoid," "middle meningeal artery," "endovascular treatment," and "embolization" [MeSH], combined with Boolean operators (“AND," "OR," and "NOT").

\section{Inclusion Criteria}

Papers written in English and published between the years 1990 and 2019 were considered eligible if they included at least one adult (70 years of age or older) with a diagnosed supratentorial CSDH who received nonsurgical primary or adjuvant treatments for CSDH. Studies needed to report on patients' characteristics, mortality, neurological outcome, recurrences, need for reintervention, and/or complications. Letters to the editor, editorials, commentaries, and literature reviews were excluded.

Two authors (J.V. and L.R.) independently conducted the first search round (abstract and titles) for eligibility and performed full-text evaluation for inclusion. Any discrepancy was solved by consensus with the senior author (P.D.B.). In order to collect the data about the ongoing clinical trials, the ClinicalTrials.gov database was consulted in November 2019.

\section{Results}

From the first literature search, we retrieved 720 articles. After the removal of duplicates and title/abstract screening for matching inclusion/exclusion criteria, 63 papers were assessed for eligibility (Fig. 1). Thirty-four of these papers were excluded for the following reasons: other reviews, case reports, unclear outcomes, or nonelderly population.

Ultimately, 29 studies were included in the data analysis: 4 were on tranexamic acid (TXA; 1 prospective randomized study, 2 retrospective trials, and 1 case report); 11 studies investigated the role of dexamethasone (DX; 3 prospective randomized controlled trials [RCTs], 2 prospective nonrandomized trials, and 6 retrospective studies; Table 1); and 14 studies investigated the role of middle meningeal artery embolization (MMAE; 6 case series, 3 case reports, 4 retrospective studies, and 1 prospective trial; Table 2).

\section{Medical Treatments: TXA and DX}

Four studies were collected on TXA, with a total of
105 cases included; ${ }^{9-12} 18$ patients received TXA as unique treatment and in 87 cases TXA was administered as adjuvant therapy following surgical drainage. Patients were enrolled based on radiological evidence of CSDHs regardless of the presence of compressive symptoms, although surgical drainage was performed in any case of severe neurological deterioration. The authors reported no adverse events related to TXA administration. In all 3 clinical trials using TXA as adjuvant or unique therapy that were included in our review, ${ }^{9-11}$ an overall reduction of hematoma volume was observed. TXA alone or as adjuvant treatment was associated with a mean reduction of the hematoma volume in all patients. In one paper, ${ }^{10} \mathrm{a}$ statistically significant difference was observed, favoring the use of TXA as adjuvant therapy after surgery.

Eleven studies investigated the role of DX. ${ }^{13-23}$ We retrieved 1067 cases in which DX was administered: in 810 cases as adjuvant treatment, whereas 257 patients received DX as primary treatment. The cohorts that received DX alone showed the most unfavorable outcomes: surgical procedures for hematoma evacuation were required, ranging from $22 \%$ to $83 \%$ of patients in different series. ${ }^{14-22}$ On the other hand, adjuvant corticosteroids after surgery resulted in a recurrence rate of $11.6 \%$, ranging from $0 \%$ to $40 \%$ among the different studies. ${ }^{16,18,19,21,23}$

\section{Endovascular Treatment: MMAE}

MMAE is a relatively new technique; it was first reported in the early 2000s. ${ }^{24}$ It was performed in 195 patients for a total of 207 procedures: in 125 cases as adjuvant treatment for recurrence after surgery or as a prophylactic measure in patients with specific risk factors, such as coagulation disorders. Conversely, MMAE was performed as the primary treatment, as an alternative to surgery, in 82 neurologically stable patients without significant compressive symptoms.

Second surgeries for hematoma recurrence were reported in 24 cases, regardless of the timing, with an overall recurrence rate of $11.6 \%$. No procedure-related complications were reported.

\section{Other Medical Treatments}

Although molecules such as angiotensin-converting enzyme (ACE) inhibitors, statins, and mannitol have been advocated by some authors, none of these agents reached a sufficient level of evidence to recommend their use. ${ }^{38-40}$

\section{Ongoing Trials}

The government database review for registered ongoing clinical trials on nonoperative management for CSDH produced 18 studies (Table 3): 4 RCTs and 1 prospective nonrandomized trial involving TXA are currently ongoing, whereas $10 \mathrm{RCT}$ are investigating the role of DX and methylprednisolone. One RCT and 2 nonrandomized clinical trials for MMAE were found and included for review.

\section{Discussion}

\section{Tranexamic Acid}

We collected data on 105 patients treated with TXA; in most of them (82.9\%) it was administered as adjuvant treat- 


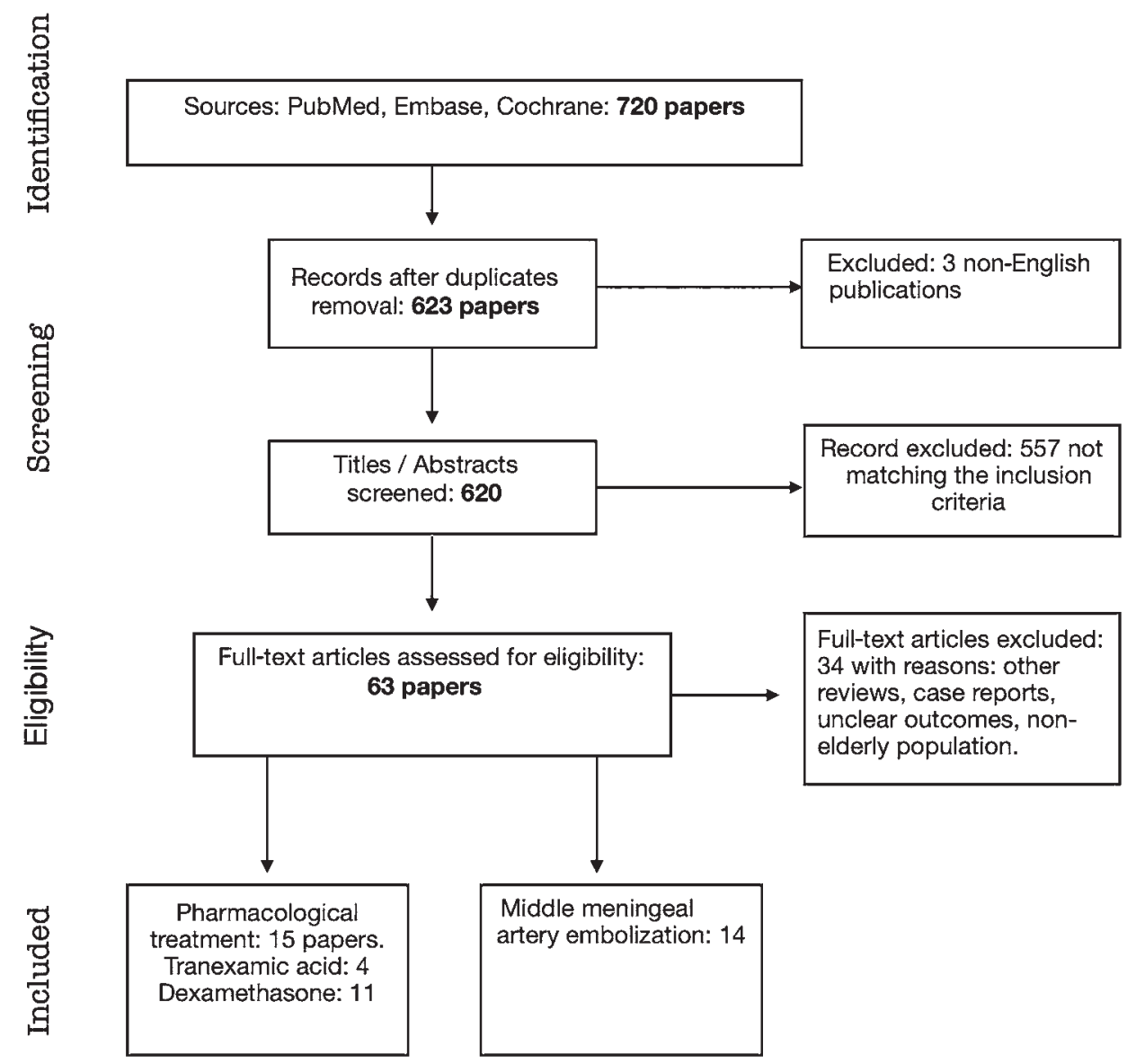

FIG. 1. PRISMA flowchart of the systematic review of studies of patients with CSDH.

ment. Hematoma volume reduction was reported in all of them, with only one recurrence and no complications. The presence of hyperfibrinolytic activities has been shown to play a major role in the pathogenesis of CSDHs. ${ }^{59,60} \mathrm{Be}-$ cause the TXA inhibits fibrinolysis and enhances the hemostasis due to antiplasmin activity, it was hypothesized that it might lead to a gradual resorption of SDH.

The level of evidence about the use of TXA is generally low (level 3b), because most of the studies (3 of 4 in our review) are retrospective or case series. Kageyama et al. ${ }^{9}$ performed the first retrospective study (level $3 \mathrm{~b}$ ) of a cohort in whom $750 \mathrm{mg}$ of TXA was administered once a day as an alternative to surgery in 18 patients, who showed a complete radiological recovery. This study was affected by its retrospective design and thus a relatively low level of evidence; moreover, patients receiving anticoagulant or antiplatelet medications, representing a wide subgroup of CSDH cases, were excluded from the study.

A higher level of evidence (level 1b) was reached by Yamada and Natori, ${ }^{10}$ who performed the first prospective RCT in a cohort of 193 patients with CSDH who were treated with a traditional burr hole for hematoma evacuation. The investigators subdivided this cohort into three groups based on adjuvant therapies: TXA, goreisan, or clinical observation. They showed no difference in the recurrence rate between surgery and TXA groups; however, the mean residual hematoma volume was significantly lower in the TXA group. No treatment-related toxicity was reported. ${ }^{9-12}$ Contraindications to TXA include comorbidities such as renal dysfunction, malignancy, cardiovascular, respiratory disease, current anticoagulant therapy, and history of thromboembolic disease, including deep vein thrombosis, pulmonary embolism, arterial thrombosis, stroke, and subarachnoid hemorrhage. ${ }^{61}$

A multicenter, double-blind, randomized phase 2B study (level $1 \mathrm{~b}$ evidence) is currently ongoing-"Tranexamic Acid in Chronic Subdural Hematomas (TRACS)" (NCT02568124) - and its two arms consist of TXA and placebo. ${ }^{42}$ According to the study design, 130 patients will be randomized to receive either $750 \mathrm{mg}$ of TXA or placebo daily, with a final follow-up at 20 weeks. Even though this study will represent the first RCT on this topic, its applicability will be affected by the exclusion of patients receiving anticoagulant medications; on the other hand, further RCTs are currently recruiting patients. ${ }^{43,44}$

Our data showed that TXA was effective for the reduction of hematoma volume in all patients, with a very low rate of recurrence (1.1\%) and no complications. In summary, while waiting for ongoing RCTs to be completed, current evidence about TXA efficacy in CSDH treatment can be considered as level $1 \mathrm{~b}$ with a grade $\mathrm{B}$ strength of recommendations. 


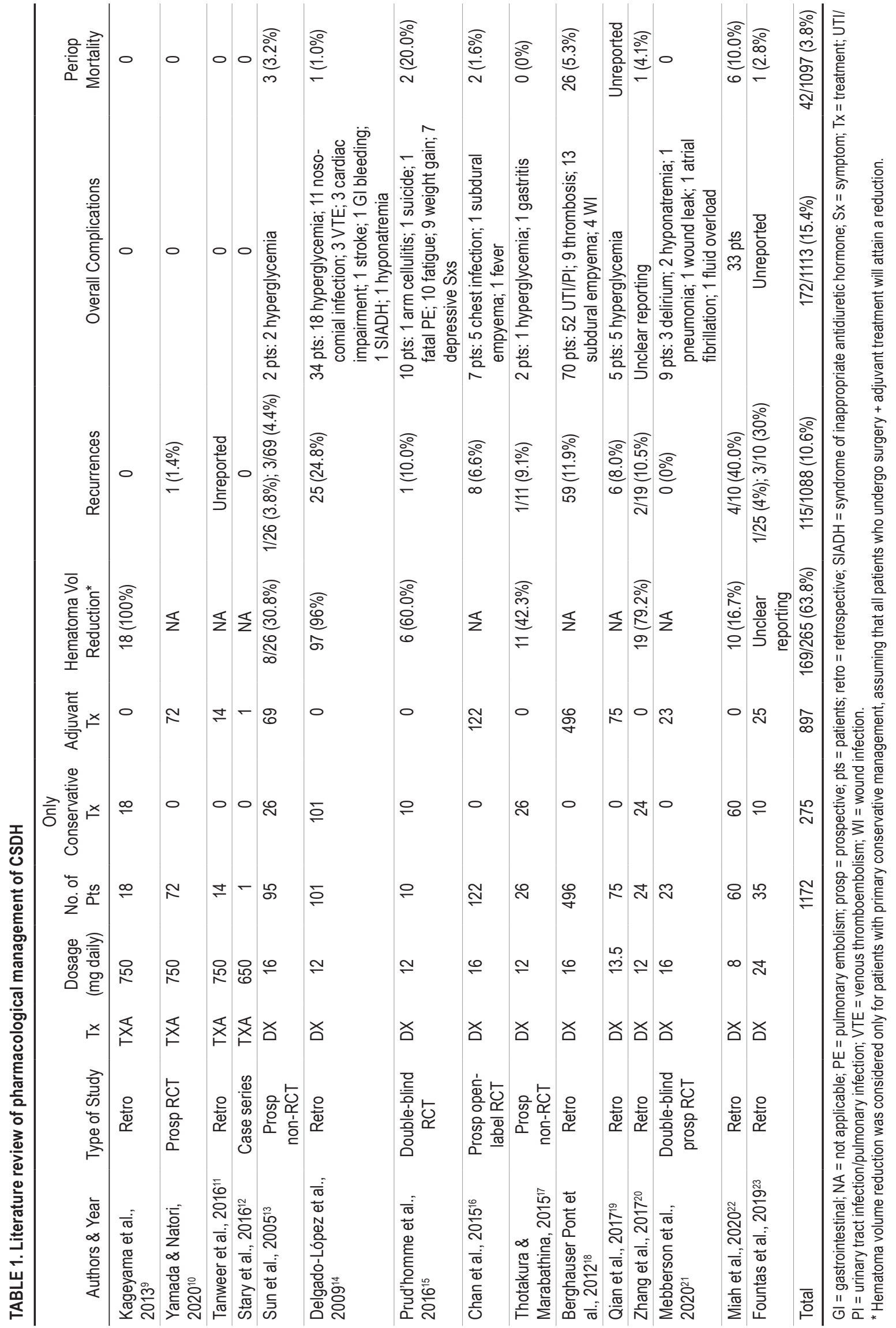


TABLE 2. MMAE in the treatment of CSDH

\begin{tabular}{|c|c|c|c|c|c|c|c|c|c|c|}
\hline $\begin{array}{c}\text { Authors \& } \\
\text { Year }\end{array}$ & Type of Study & $\begin{array}{c}\text { No. of } \\
\text { Pts/CSDHs }\end{array}$ & $\begin{array}{l}\text { Embolic } \\
\text { Material }\end{array}$ & $\begin{array}{c}\text { Primary } \\
\text { Tx }\end{array}$ & $\begin{array}{l}\text { Adjuvant } \\
\text { Tx }\end{array}$ & $\begin{array}{c}\text { Hematoma Vol } \\
\text { Reduction* }\end{array}$ & $\begin{array}{l}\text { Recurrences } \\
\text { After MMAE }\end{array}$ & $\begin{array}{l}\text { Complications } \\
\text { of MMAE }\end{array}$ & Mortality & Follow-Up \\
\hline $\begin{array}{l}\text { Mandai et } \\
\text { al., } 2000^{24}\end{array}$ & Case report & 1 & PC & 0 & 1 & NA & 0 & 0 & 0 & $7 \mathrm{mos}$ \\
\hline $\begin{array}{l}\text { Hirai et al., } \\
2004^{25}\end{array}$ & Case report & 2 & $\begin{array}{l}\text { PC, } \\
\text { PVA }\end{array}$ & 0 & 2 & NA & 0 & 0 & 0 & $9 \mathrm{mos}$ \\
\hline $\begin{array}{l}\text { Ishihara et } \\
\text { al., } 2007^{26}\end{array}$ & Case series & 7 & NBCA & 0 & 7 & NA & 0 & 0 & 0 & $15 \mathrm{mos}$ \\
\hline $\begin{array}{l}\text { Mino et al., } \\
2010^{27}\end{array}$ & Case series & 4 & PC & 0 & 4 & NA & $2(50.0 \%)$ & 0 & 0 & $6 \mathrm{mos}$ \\
\hline $\begin{array}{l}\text { Hashimoto } \\
\text { et al., } 2013^{28}\end{array}$ & Case series & 5 & $\begin{array}{l}\text { NBCA, } \\
\text { PVA }\end{array}$ & 0 & 5 & NA & 0 & 0 & 0 & Unreported \\
\hline $\begin{array}{l}\text { Chihara et } \\
\text { al., } 2014^{29}\end{array}$ & Case report & 3 & $\begin{array}{l}\text { PC, } \\
\text { PVA }\end{array}$ & 0 & 3 & NA & $1(33.3 \%)$ & 0 & 0 & $2 \mathrm{yrs}$ \\
\hline $\begin{array}{l}\text { Tempaku et } \\
\text { al., } 2015^{30}\end{array}$ & Case series & 5 & PVA & 0 & 5 & NA & $4(80.0 \%)$ & 0 & 0 & $6-60$ wks \\
\hline Kim, $2017^{31}$ & Retro & 20 & PVA & 0 & 20 & NA & $1(5.0 \%)$ & 0 & $2(10.0 \%)$ & 6 mos \\
\hline $\begin{array}{l}\text { Matsumoto } \\
\text { et al., } 2018^{32}\end{array}$ & Retro & 4 & $\begin{array}{l}\text { NCBA, } \\
\text { PC }\end{array}$ & 0 & 4 & NA & 0 & 0 & 0 & $3-6$ mos \\
\hline $\begin{array}{l}\text { Link et al., } \\
2018^{33}\end{array}$ & Case series & $6 / 7$ & PVA & 0 & 7 & NA & $1 / 7$ & 0 & 0 & Unreported \\
\hline $\begin{array}{l}\text { Link et al., } \\
2019^{34}\end{array}$ & Case series & $39 / 50$ & PVA & 50 & 0 & $31 / 50(62.0 \%)$ & $4(8.0 \%)$ & 0 & 0 & 6 wks \\
\hline $\begin{array}{l}\text { Ban et al., } \\
2018^{35}\end{array}$ & $\begin{array}{c}\text { Prosp } \\
\text { nonrandomized }\end{array}$ & 72 & $\begin{array}{l}\text { PVA, } \\
\text { PC }\end{array}$ & 27 & 45 & $27 / 27(100 \%)$ & $1 / 45(2.2 \%), 0 / 27$ & 0 & 0 & $6 \mathrm{mos}$ \\
\hline $\begin{array}{l}\text { Farkas, } \\
2018^{36}\end{array}$ & Retro & 10 & PVA & 5 & 5 & $3 / 5(60.0 \%)$ & 0 & 0 & 0 & $1 \mathrm{yr}$ \\
\hline $\begin{array}{l}\text { Okuma et } \\
\text { al., } 2019^{37}\end{array}$ & Retro & 17 & NCBA & 0 & 17 & NA & 0 & 0 & 0 & Unreported \\
\hline Total & & $195 / 207$ & & 82 & 125 & $61 / 82(74.4 \%)$ & $24 / 207$ (11.6\%) & 0 & $2(1.0 \%)$ & \\
\hline
\end{tabular}

$\mathrm{NBCA}=\mathrm{N}$-butyl cyanoacrylate PC = platinum coils; $\mathrm{PVA}=$ polyvinyl alcohol.

* Hematoma volume reduction was considered only for patients with primary conservative management, assuming that all patients who undergo surgery + adjuvant treatment will attain a reduction.

\section{Dexamethasone}

We collected data in 1067 patients treated with DX, 257 as only conservative treatment and 810 as adjuvant (Table 1). Of the patients with primary conservative management, $61.1 \%$ attained hematoma volume reduction, whereas the overall rate for recurrences (primary and adjuvant treatment) was $11.6 \%$ (Table 4). Conversely, of the 1008 patients in whom the presence or absence of complications was reported, 172 patients (17.1\%) had complications (Tables 1 and 4).

Although multifactorial mechanisms are involved in CSDH development, its inflammatory etiology has already been proposed and is being increasingly accepted. ${ }^{62-64}$ Corticosteroids, through the inhibition of these inflammatory and angiogenetic factors, could slow down the CSDH growth and even determine its resorption.

In the 1970s, Bender and Christoff ${ }^{65}$ were the first to evaluate the efficacy of DX for CSDH in a clinical setting and suggested its use as treatment in neurologically stable patients.

Sun et al. ${ }^{13}$ performed a prospective study (level $2 \mathrm{~b}$ evi- dence) on a cohort of 108 patients, in which 26 patients were treated with DX alone, 69 underwent burr-hole craniotomy and adjuvant corticosteroids, and 13 were treated with surgical drainage alone. In the corticosteroid group, 1 patient required surgical drainage, whereas in the surgical group hematoma recurrence was reported in 3 patients; no significant difference between the two groups was measured.

In 2009, Delgado-López et al. ${ }^{14}$ performed a retrospective study (level $3 \mathrm{~b}$ evidence) including a cohort of 120 patients. Nineteen patients underwent surgery, whereas 101 were treated with DX alone. Again, no significant differences between the two groups were found.

A pilot RCT (level $2 \mathrm{~b}$ evidence) by Prud'homme et al. ${ }^{15}$ enrolled 10 patients affected by CSDH who were treated with DX and 10 patients who received placebo. The authors reported no statistically significant differences between the two groups, although the study was prematurely terminated due to a high incidence of complications in the DX group.

Berghauser Pont and colleagues ${ }^{18}$ reported a cohort 
TABLE 3. Ongoing clinical trials of nonsurgical treatments for CSDH

\begin{tabular}{|c|c|c|c|c|c|c|}
\hline Trial No. & Therapy & Type of Study & No. of Pts & Current Status & Estimated Completion Date & Country \\
\hline NCT0328021241 & TXA & Randomized & 60 & Unknown & March 2018 & Canada \\
\hline NCT0256812442 & TXA & Randomized & 130 & Unknown & June 2019 & Canada \\
\hline NCT0335325943 & TXA & Randomized & 600 & Recruiting & November 2020 & Norway \\
\hline NCT0358229344 & TXA & Randomized & 130 & Recruiting & December 2021 & Netherlands \\
\hline NCT02618382 45 & TXA & Nonrandomized & 50 & Unknown & January 2018 & USA \\
\hline NCT02938468 46 & $\mathrm{DX}$ & Randomized & 326 & Recruiting & September 2021 & Canada \\
\hline NCT0211178547 & $\mathrm{DX}$ & Randomized & 10 & Terminated & December 2018 & USA \\
\hline NCT0219232048 & $\mathrm{DX}$ & Randomized & 60 & Unknown & September 2015 & China \\
\hline NCT0236232149 & DX & Randomized & 20 & Terminated & February 2016 & Canada \\
\hline ACTRN1261300017577450 & DX & Randomized & Unknown & Ongoing & Unknown & Australia \\
\hline NCT0138002851 & $\mathrm{DX}$ & Randomized & 340 & Active & Unknown & France \\
\hline NCT0265060952 & MPSS & Randomized & 202 & Recruiting & Unknown & France \\
\hline EudraCT2011-003544-4253 & $\mathrm{DX}$ & Randomized & 790 & Ongoing & Unknown & Austria \\
\hline EudraCT2014-004948-3554 & DX & Randomized & 750 & Terminated & August 2019 & UK \\
\hline EudraCT2015-001563-3955 & DX & Randomized & 170 & Ongoing & Unknown & Netherlands \\
\hline NCT0330739556 & MMAE & Nonrandomized & 50 & Recruiting & December 2021 & USA \\
\hline NCT0406511357 & MMAE & Nonrandomized & 600 & Recruiting & January 2022 & USA \\
\hline NCT0409581958 & MMAE & Randomized & 50 & Recruiting & April 2022 & USA \\
\hline
\end{tabular}

MPSS $=$ methylprednisolone.

(level 3b evidence) of 496 patients treated with adjuvant DX following surgical drainage, and the hematoma recurrence rate was $11.9 \%$. In the literature, the risk of recurrence is estimated at $10 \% .{ }^{66}$

Almenawer et al. ${ }^{5}$ published a meta-analysis (level 1a evidence) of 34,829 patients with CSDH. The use of steroids in a pooled analysis from 5 nonrandomized studies did not result in outcome differences when compared with surgical management. Meta-analyses of 17 pooled cohorts resulted in no evidence supporting favorable outcomes when using steroids in addition to surgeries; however, there were higher rates of morbidity. These findings are comparable to our data. On the other hand, another sys-

TABLE 4. Summary of the main data collected on conservative treatments compared to data collected by a systematic review of surgical treatment

\begin{tabular}{lcccc}
\hline \multicolumn{1}{c}{ Parameter } & TXA & DX & MMAE & Surgery $^{*}$ \\
\hline No. of studies & \multicolumn{1}{c}{4} & 11 & 14 & 16 \\
\hline No. of pts & 105 & 1067 & 195 & 1407 \\
\hline Primary Tx & $17.1 \%$ & $24.1 \%$ & $39.6 \%$ & NA \\
\hline Adjuvant Tx & $82.9 \%$ & $75.9 \%$ & $60.4 \%$ & NA \\
\hline Hematoma vol reductiont & $100 \%$ & $61.1 \%$ & $74.4 \%$ & $82.0 \%$ \\
\hline Recurrence & $1.1 \%$ & $11.6 \%$ & $11.6 \%$ & $11.0 \%$ \\
\hline Overall complications & $0 \%$ & $17.1 \%$ & $0 \%$ & $11.0 \%$ \\
\hline Mortality & $0 \%$ & $4.2 \%$ & $1.0 \%$ & $4.0 \%$ \\
\hline
\end{tabular}

* Data from RCTs collected by Almenawer et al. ${ }^{5}$

$\dagger$ Hematoma volume reduction was considered only for patients with primary conservative management, assuming that all patients who undergo surgery + adjuvant treatment will attain a reduction. tematic review (level 1a evidence) by Holl et al. ${ }^{67}$ specifically evaluating corticosteroid treatment compared with surgery in CSDH suggested that the addition of steroids to surgery might be effective in terms of need for reintervention and mortality rate. Indeed, the authors also warned that their results must be interpreted with caution in light of the serious risk of bias of the included studies.

Finally, Mebberson et al. ${ }^{21}$ published a prospective RCT (level $1 \mathrm{~b}$ evidence) including 47 patients, 23 assigned to DX treatment and 24 to placebo after traditional surgery. They found a weak statistical significance $(\mathrm{p}=0.049)$ in comparing the two groups; the hematoma recurrence rate was $20.83 \%$ in the placebo group and $0 \%$ in the DX group.

Due to the paucity of level 1 evidence, several RCTs were initiated in the last few years (Table 3). Steroids are evaluated as treatment in addition to surgery or as therapy alone versus surgery. ${ }^{68}$ Our data showed that the use of DX for CSDHs is still questionable. As the primary treatment, it is absolutely noneffective; as adjuvant treatment, in $61.1 \%$ of patients DX caused hematoma volume reduction. On the other hand, more than $15 \%$ of patients had complications and the recurrence rate is comparable to that of surgery alone (Table 4).

Currently, level 1 evidence coming from systematic reviews ${ }^{5}$ and prospective $\mathrm{RCTs}^{21}$ has reached contradictory conclusions on the safety and efficacy of DX in CSDH treatment. The strength of recommendations for its use could be considered as grade C. Many RCTs are nearing completion and, if successful, will probably answer whether DX could represent a useful adjuvant treatment after surgery for reducing risks for recurrence.$^{67}$ However, patient selection should be meticulously consideredavoiding those suffering from diabetes mellitus, acute or chronic infections, and hypertension. 


\section{Middle Meningeal Artery Embolization}

We collected data in 195 patients treated with MMAE for a total of 207 procedures, 82 as only primary treatment and 125 as adjuvant (Table 2). Hematoma volume reduction was reported in $74.4 \%$ of patients, with recurrence in $11.6 \%$. No procedure-related complications were reported.

An irregular wispy appearance of the MMA at angiography has been reported in $\mathrm{CSDH},{ }^{35}$ due to the presence of dysplastic vessels and capillary webs. The possibility to directly visualize this abnormal vasculature fostered the idea for an endovascular treatment through the catheterization and embolization of MMA. Recently, a systematic review of this technique ${ }^{69}$ (level 3 evidence) showed that currently data only exist from case series and nonrandomized studies with low numbers, and MMAE has mostly been applied to recurrent CSDH.

Ban et al ${ }^{35}$ performed the only available prospective study (level $2 \mathrm{~b}$ evidence). They enrolled 72 patients undergoing MMAE (27 patients with MMAE alone and 45 patients with MMAE after surgery) and compared their results with a historical cohort of 402 surgically treated cases. They concluded that MMAE was more effective than traditional treatment because they reported no treatment failure or complications related to the endovascular procedure. However, although their results are encouraging, the study design negatively influenced the level of evidence, thus leaving any eventual data confirmation and conclusion to properly designed clinical trials.

MMAE has also been hypothesized to improve outcome in patients treated with antithrombotic drugs. In fact, the Embolization of the Middle Meningeal Artery in Chronic Subdural Hematoma (EMMACS) study ${ }^{70}$ is assessing early resumption of anticoagulants following surgery with and without MMAE.

Our data showed that MMAE is a relatively safe and effective procedure, in particular for recurrences, although data regarding related adverse effects, such as intracerebral hemorrhages, vasospasms, and strokes, are vague and not fully assessed; thus, the effective complication rate could have been underestimated. The first RCT is currently recruiting patients ${ }^{58}$ in the US and is estimated to be completed in 2022. In conclusion, from literature evaluation for MMAE in CSDH we can consider level 3 evidence with grade B strength of recommendation.

In Table 4 we have summarized the different outcomes for conservative treatments compared with surgery.

\section{Limitations of the Study}

In most of the patients the evaluated treatments were given as adjuvants after surgery; this could affect the evaluation of the real efficacy of each treatment. One of the major limitations was the poor level of evidence of several of the collected studies, which could represent a bias in the correct evaluation of the extracted data. Moreover, an expected limitation of including resources with variable qualities, definitions, follow-ups, and diagnostic criteria is the inevitable heterogeneity detected in some outcomes.

Another limitation of this study is the relatively small number of patients due to selective inclusion of elderly patients. Unfortunately, due to the restricted number of data provided by studies, detailed differences among manage- ment options, including variable minor techniques, different management, and different medications, were not evaluated in this study.

\section{Conclusions}

Recurrences, reoperations, and complications represent heavy burdens for patients older than 70 years of age and suffering from CSDH. TXA was shown to be effective for the reduction of hematoma volume in all patients, with a very low rate of recurrence and no complications. The use of DX remains questionable. As a primary treatment, it is absolutely ineffective; as an adjuvant treatment, it can cause hematoma volume reduction but with a risk of complications of more than $15 \%$ and a recurrence rate comparable to that of surgery alone. MMAE represents an interesting endovascular solution as an adjuvant treatment in CSDH recurrences. Even though few reports are available, our data showed that it is safe and effective, in particular for recurrences. Whereas surgery is still considered the gold standard treatment in cases of neurological impairment, the aforementioned alternatives should be considered in carefully selected patients. In order to improve outcomes, a tailored, personalized therapy should be sought. Patients could be stratified for operative versus conservative treatment based on the need for mass effect removal. Furthermore, adjuvant therapies could be proposed based on the risk of recurrence and complications. Results from clinical trials are needed to confirm these preliminary data and better identify any patient subgroups benefiting the most from each of them.

\section{References}

1. Kudo H, Kuwamura K, Izawa I, et al. Chronic subdural hematoma in elderly people: present status on Awaji Island and epidemiological prospect. Neurol Med Chir (Tokyo). 1992; 32(4):207-209.

2. Karibe H, Kameyama M, Kawase M, et al. Epidemiology of chronic subdural hematomas. Article in Japanese. No Shinkei Geka. 2011;39(12):1149-1153.

3. Asghar M, Adhiyaman V, Greenway MW, et al. Chronic subdural haematoma in the elderly-a North Wales experience. $J$ $R$ Soc Med. 2002;95(6):290-292.

4. Toi H, Kinoshita K, Hirai S, et al. Present epidemiology of chronic subdural hematoma in Japan: analysis of 63,358 cases recorded in a national administrative database. $J \mathrm{Neu}$ rosurg. 2018;128(1):222-228.

5. Almenawer SA, Farrokhyar F, Hong C, et al. Chronic subdural hematoma management: a systematic review and metaanalysis of 34,829 patients. Ann Surg. 2014;259(3):449-457.

6. Liu W, Bakker NA, Groen RJ. Chronic subdural hematoma: a systematic review and meta-analysis of surgical procedures. $J$ Neurosurg. 2014;121(3):665-673.

7. Uno M, Toi H, Hirai S. Chronic subdural hematoma in elderly patients: is this disease benign? Neurol Med Chir (Tokyo). 2017;57(8):402-409.

8. Moher D, Liberati A, Tetzlaff J, Altman DG. Preferred reporting items for systematic reviews and meta-analyses: the PRISMA statement. Int J Surg. 2010;8(5):336-341.

9. Kageyama H, Toyooka T, Tsuzuki N, Oka K. Nonsurgical treatment of chronic subdural hematoma with tranexamic acid. J Neurosurg. 2013;119(2):332-337.

10. Yamada T, Natori Y. Prospective study on the efficacy of orally administered tranexamic acid and Goreisan for the 
prevention of recurrence after chronic subdural hematoma burr hole surgery. World Neurosurg. 2020;134:e549-e553.

11. Tanweer O, Frisoli FA, Bravate C, et al. Tranexamic acid for treatment of residual subdural hematoma after bedside twistdrill evacuation. World Neurosurg. 2016;91:29-33.

12. Stary JM, Hutchins L, Vega RA. Tranexamic acid for recurring subdural hematomas following surgical evacuation: a clinical case series. J Neurol Surg A Cent Eur Neurosurg. 2016;77(5):422-426.

13. Sun TFD, Boet R, Poon WS. Non-surgical primary treatment of chronic subdural haematoma: preliminary results of using dexamethasone. Br J Neurosurg. 2005;19(4):327-333.

14. Delgado-López PD, Martín-Velasco V, Castilla-Díez JM, et al. Dexamethasone treatment in chronic subdural haematoma. Neurocirugia (Astur). 2009;20(4):346-359.

15. Prud'homme M, Mathieu F, Marcotte N, Cottin S. A pilot placebo controlled randomized trial of dexamethasone for chronic subdural hematoma. Can J Neurol Sci. 2016;43(2): 284-290.

16. Chan DYC, Sun TFD, Poon WS. Steroid for chronic subdural hematoma? A prospective phase IIB pilot randomized controlled trial on the use of dexamethasone with surgical drainage for the reduction of recurrence with reoperation. Chin Neurosurg J. 2015;1:2.

17. Thotakura AK, Marabathina NR. Nonsurgical treatment of chronic subdural hematoma with steroids. World Neurosurg. 2015;84(6):1968-1972.

18. Berghauser Pont LM, Dammers R, Schouten JW, et al. Clinical factors associated with outcome in chronic subdural hematoma: a retrospective cohort study of patients on preoperative corticosteroid therapy. Neurosurgery. 2012;70(4): 873-880.

19. Qian Z, Yang D, Sun F, Sun Z. Risk factors for recurrence of chronic subdural hematoma after burr hole surgery: potential protective role of dexamethasone. Br J Neurosurg. 2017;31(1): 84-88.

20. Zhang Y, Chen S, Xiao Y, Tang W. Effects of dexamethasone in the treatment of recurrent chronic subdural hematoma. World Neurosurg. 2017;105:115-121.

21. Mebberson K, Colditz M, Marshman LAG, et al. Prospective randomized placebo-controlled double-blind clinical study of adjuvant dexamethasone with surgery for chronic subdural haematoma with post-operative subdural drainage: interim analysis. J Clin Neurosci. 2020;71:153-157.

22. Miah IP, Herklots M, Roks G, et al. Dexamethasone therapy in symptomatic chronic subdural hematoma (DECSA-R): a retrospective evaluation of initial corticosteroid therapy versus primary surgery. J Neurotrauma. 2020;37(2):366-372.

23. Fountas K, Kotlia P, Panagiotopoulos V, et al. The outcome after surgical vs nonsurgical treatment of chronic subdural hematoma with dexamethasone. Interdiscip Neurosurg. 2019; 16:70-74.

24. Mandai S, Sakurai M, Matsumoto Y. Middle meningeal artery embolization for refractory chronic subdural hematoma. Case report. J Neurosurg. 2000;93(4):686-688.

25. Hirai S, Ono J, Odaki M, et al. Embolization of the middle meningeal artery for refractory chronic subdural haematoma. Usefulness for patients under anticoagulant therapy. Interv Neuroradiol. 2004;10(suppl 2):101-104.

26. Ishihara H, Ishihara $\mathrm{S}$, Kohyama $\mathrm{S}$, et al. Experience in endovascular treatment of recurrent chronic subdural hematoma. Interv Neuroradiol. 2007;13(suppl 1):141-144.

27. Mino M, Nishimura S, Hori E, et al. Efficacy of middle meningeal artery embolization in the treatment of refractory chronic subdural hematoma. Surg Neurol Int. 2010;1:78.

28. Hashimoto T, Ohashi T, Watanabe D, et al. Usefulness of embolization of the middle meningeal artery for refractory chronic subdural hematomas. Surg Neurol Int. 2013;4:104.

29. Chihara H, Imamura H, Ogura T, et al. Recurrence of a re- fractory chronic subdural hematoma after middle meningeal artery embolization that required craniotomy. NMC Case Rep J. 2014;1(1):1-5.

30. Tempaku A, Yamauchi S, Ikeda H, et al. Usefulness of interventional embolization of the middle meningeal artery for recurrent chronic subdural hematoma: five cases and a review of the literature. Interv Neuroradiol. 2015;21(3):366-371.

31. Kim E. Embolization therapy for refractory hemorrhage in patients with chronic subdural hematomas. World Neurosurg. 2017;101:520-527.

32. Matsumoto H, Hanayama H, Okada T, et al. Which surgical procedure is effective for refractory chronic subdural hematoma? Analysis of our surgical procedures and literature review. J Clin Neurosci. 2018;49:40-47.

33. Link TW, Schwarz JT, Paine SM, et al. Middle meningeal artery embolization for recurrent chronic subdural hematoma: a case series. World Neurosurg. 2018;118:e570-e574.

34. Link TW, Boddu S, Paine SM, et al. Middle meningeal artery embolization for chronic subdural hematoma: a series of 60 cases. Neurosurgery. 2019;85(6):801-807.

35. Ban SP, Hwang G, Byoun HS, et al. Middle meningeal artery embolization for chronic subdural hematoma. Radiology. 2018;286(3):992-999.

36. Farkas J. Radiographic efficacy of middle meningeal artery embolization in treatment of chronic subdural hematoma. Interv Neuroradiol. 2018;24(4):455-462.

37. Okuma Y, Hirotsune N, Sato Y, et al. Midterm follow-up of patients with middle meningeal artery embolization in intractable chronic subdural hematoma. World Neurosurg. 2019; 126:e671-e678.

38. Wang D, Li T, Tian Y, et al. Effects of atorvastatin on chronic subdural hematoma: a preliminary report from three medical centers. J Neurol Sci. 2014;336(1-2):237-242.

39. Li T, Wang D, Tian Y, et al. Effects of atorvastatin on the inflammation regulation and elimination of subdural hematoma in rats. J Neurol Sci. 2014;341(1-2):88-96.

40. Poulsen FR, Munthe S, Søe M, Halle B. Perindopril and residual chronic subdural hematoma volumes six weeks after burr hole surgery: a randomized trial. Clin Neurol Neurosurg. 2014; 123:4-8.

41. Tranexamic Acid in the Treatment of Residual Chronic Subdural Hematoma (TRACE). NCT03280212. Accessed August 6, 2020. https://clinicaltrials.gov/ct2/show/NCT03280212

42. Tranexamic Acid in Chronic Subdural Hematomas (TRACS). NCT02568124. Accessed August 6, 2020. https:// clinicaltrials.gov/ct2/show/NCT02568124

43. Tocilizumab (RoActemra) and Tranexamic Acid (Cyklokapron) Used as Adjuncts to Chronic Subdural Hematoma Surgery. NCT03353259. Accessed August 6, 2020. https:// clinicaltrials.gov/ct2/show/NCT03353259

44. Tranexamic Acid to Prevent OpeRation in Chronic Subdural Hematoma (TORCH). NCT03582293. Accessed August 6, 2020. https://clinicaltrials.gov/ct2/show/NCT03582293

45. A Study on the Safety of Tranexamic Acid for the Chronic Subdural Hematoma Population. NCT02618382. Accessed August 6, 2020. https://clinicaltrials.gov/ct2/show/ NCT02618382

46. Mgt of Chronic Subdural Hematoma Using Dexamethasone. NCT02938468. Accessed August 6, 2020. https:// clinicaltrials.gov/ct2/show/NCT02938468

47. Dexamethasone Versus Burr Hole Craniostomy for Symptomatic Chronic Subdural Hematoma (DECS). NCT02111785. Accessed August 6, 2020. https://clinicaltrials. gov/ct2/show/NCT02111785

48. The Clinical Study of Atorvastatin and Dexamethasone on Treatment for Chronic Subdural Hematoma in the Patients With Coagulation Disorders. NCT02192320. Accessed August 6, 2020. https://clinicaltrials.gov/ct2/show/NCT02192320

49. Role of Dexamethasone in the Conservative Treatment of 
Chronic Subdural Hematoma. NCT02362321. Accessed August 6, 2020. https://clinicaltrials.gov/ct2/show/NCT02362321

50. A Study to Compare Surgery for Chronic Subdural Haematoma With and Without Corticosteroids. ACTRN12613000175774. Accessed August 6, 2020. https://www.anzctr.org.au/Trial/Registration/TrialReview. aspx?id=363683

51. Interest of Oral Corticosteroids in the Treatment of Chronic Subdural Hematomas (hemacort). NCT01380028. Accessed August 6, 2020. https://clinicaltrials.gov/ct2/show/ NCT01380028

52. Treatment of Chronic Subdural Hematoma by Corticosteroids (SUCRE). NCT02650609. Accessed August 6, 2020. https://clinicaltrials.gov/ct2/show/NCT02650609

53. DRES-A Prospective, Multi-Center, Double Blind, Randomized, Placebo Controlled Study to Assess the Efficacy of Dexamethason in Reducing the Reoperation Rate in Patients with Chronic Subdural Hematoma. EudraCT2011-003544-42. Accessed August 6, 2020. https://www.clinicaltrialsregister.eu/ctr-search/ trial/2011-003544-42/AT

54. A Randomised Blinded Trial of a Steroid (Dexamethasone) Compared to a Placebo for Adult Patients Who Have Suffered a Chronic Bleed on the Surface of the Brain. EudraCT2014-004948-35. Accessed August 6, 2020. https://www.clinicaltrialsregister.eu/ctr-search/ trial/2014-004948-35/GB

55. Dexamethasone (DXM) Therapy in Symptomatic Patients With Chronic Subdural Hematoma (DECSA-Trial). Effect of Initial Corticosteroid Therapy Versus Primary Surgery on Clinical Outcome. EudraCT2015-001563-39. Accessed August 6, 2020. https://www.clinicaltrialsregister.eu/ctr-search/ trial/2015-001563-39/NL

56. Middle Meningeal Artery Embolization for Treatment of Chronic Subdural Hematoma. NCT03307395. Accessed August 6, 2020. https://clinicaltrials.gov/ct2/show/NCT03307395

57. Middle Meningeal Artery Embolization for Chronic Subdural Hematoma. NCT04065113. Accessed August 6, 2020. https://clinicaltrials.gov/ct2/show/NCT04065113

58. Middle Meningeal Artery (MMA) Embolization Compared to Traditional Surgical Strategies to Treat Chronic Subdural Hematomas (cSDH). NCT04095819. Accessed August 6, 2020. https://clinicaltrials.gov/ct2/show/NCT04095819

59. Fujisawa $\mathrm{H}$, Ito $\mathrm{H}$, Kashiwagi $\mathrm{S}$, et al. Kallikrein-kinin system in chronic subdural haematomas: its roles in vascular permeability and regulation of fibrinolysis and coagulation. $J$ Neurol Neurosurg Psychiatry. 1995;59(4):388-394.

60. Harada K, Orita T, Abiko S, Aoki H. Coagulation and fibrinolysis in chronic subdural hematoma. Measurement of fibrinopeptides. Article in Japanese. Neurol Med Chir (Tokyo). 1989;29(2):113-116.

61. Bala HR, Lee S, Wong C, et al. Oral tranexamic acid for the treatment of melasma: a review. Dermatol Surg. 2018;44(6): 814-825.

62. Holl DC, Volovici V, Dirven CMF, et al. Pathophysiology and nonsurgical treatment of chronic subdural hematoma: from past to present to future. World Neurosurg. 2018;116:402-411. e2.
63. Edlmann E, Giorgi-Coll S, Whitfield PC, et al. Pathophysiology of chronic subdural haematoma: inflammation, angiogenesis and implications for pharmacotherapy. J Neuroinflammation. 2017;14(1):108.

64. Hara M, Tamaki M, Aoyagi M, Ohno K. Possible role of cyclooxygenase-2 in developing chronic subdural hematoma. $J$ Med Dent Sci. 2009;56(3):101-106.

65. Bender MB, Christoff N. Nonsurgical treatment of subdural hematomas. Arch Neurol. 1974;31(2):73-79.

66. Kolias AG, Chari A, Santarius T, Hutchinson PJ. Chronic subdural haematoma: modern management and emerging therapies. Nat Rev Neurol. 2014;10(10):570-578.

67. Holl DC, Volovici V, Dirven CMF, et al. Corticosteroid treatment compared with surgery in chronic subdural hematoma: a systematic review and meta-analysis. Acta Neurochir (Wien). 2019;161(6):1231-1242.

68. Edlmann E, Holl DC, Lingsma HF, et al. Systematic review of current randomised control trials in chronic subdural haematoma and proposal for an international collaborative approach. Acta Neurochir (Wien). 2020;162(4):763-776.

69. Waqas M, Vakhari K, Weimer PV, et al. Safety and effectiveness of embolization for chronic subdural hematoma: systematic review and case series. World Neurosurg. 2019;126: $228-236$

70. Iorio-Morin C, Touchette C, Lévesque M, et al. Chronic subdural hematoma: toward a new management paradigm for an increasingly complex population. J Neurotrauma. 2018; 35(16):1882-1885.

\section{Disclosures}

The authors report no conflict of interest concerning the materials or methods used in this study or the findings specified in this paper.

\section{Author Contributions}

Conception and design: Scerrati, Visani, Cavallo, De Bonis. Acquisition of data: Visani, Ricciardi, Dones. Analysis and interpretation of data: Visani, Rustemi. Drafting the article: Scerrati, Ricciardi. Critically revising the article: Scerrati, Dones, Rustemi, Cavallo, De Bonis. Reviewed submitted version of manuscript: Scerrati, Rustemi, Cavallo, De Bonis. Approved the final version of the manuscript on behalf of all authors: Scerrati. Administrative/technical/material support: Ricciardi, Dones, Cavallo. Study supervision: Scerrati, De Bonis.

\section{Correspondence}

Alba Scerrati: Sant'Anna University Hospital, Ferrara, Italy. a.scerrati@gmail.com. 